\title{
A Review of Field Electron Source Use in Electron Microscopes
}

\author{
By L. W. Swanson and G.A. Schwind \\ FEI Company, 5350 NE Dawson Creek Dr., Hillsboro, OR 97124, USA
}

During the past 35 years the principal electron source used in scanning electron microscopes (SEM) and transmission electron microscopes (TEM) has transitioned from the thermionic to the field electron (FE) cathode. This transition has allowed improved performance of electron microscopes such that atomic resolution is now routinely achieved in TEM and scanning transmission (STEM) instruments. For the SEM the use of FE sources has allowed sub-one nanometer performance over a wide range of beam voltages. This presentation will review the key characteristics of the various FE sources now in use and prospects for improvement.

In principal FE sources can be operated in a continuum of modes as illustrated in figure 1. The current density $J(F, T, \phi)$ is a function of the applied electric field $F$, the source temperature $\mathrm{T}$ and the work function $\phi$. The two extremes of the continuum illustrated in figure 1 are referred to as the cold field emission (CFE) mode for $F>F_{2}$ where all electrons tunnel through the potential barrier and the Schottky emission (SE) mode for $F$ $<\mathrm{F}_{1}$, where most electrons are thermally excited over the potential barrier. The function $\mathrm{J}(\mathrm{F}, \mathrm{T}, \phi)$ is such that if a constant $\mathrm{J}$ is desired across the continuum of emission modes the low field $\mathrm{SE}$ mode requires a combination of high $\mathrm{T}$ and low $\mathrm{F}$ whereas the $\mathrm{CFE}$ mode requires a combination of high $\mathrm{F}$ and low $\mathrm{T}$. Depending on the source material, degree of vacuum and other environmental factors, a particular FE source may not exhibit stable emission in one or more of the possible emission modes.

For the CFE mode the source is typically more sensitive to the vacuum environment and thus requires $\mathrm{P}=10^{-10}$ torr. On the other hand for the SE mode of operation some sources can operate with a high degree of stability for $\mathrm{P}=5 \times 10^{-9}$ torr. For all the emission modes $\mathrm{J}$ is exponentially related to $\mathrm{F}$ and $\phi$ which means that both must remain constant with time in order to achieve a stable J. Since $F=f(r, \alpha) V_{o}$, where $r, \alpha$, and $V_{o}$ are the emitter radius, cone angle and anode voltage respectively, it is imperative that both $\phi$ and the emitter micro-geometry remain constant with time. This is the primary challenge for the design of a FE source that can function in a commercial instrument.

A viable FE source for use in a high resolution SEM or TEM must also exhibit a high reduced axial brightness $\mathrm{B}_{\mathrm{r}}$ and a low energy spread (usually referred to as the full width at half maximum (FWHM) of the total energy distribution illustrated in figure1). The beam current in an SEM is directly proportional to $\mathrm{B}_{\mathrm{r}}$ and the FWHM value contributes to the focused beam size via the chromatic aberration. Figure 2 gives the theoretical values of the FWHM vs. T at various values of $\phi$ at constant J over the continuum of emission modes. It is clear that to operate a source in the SE mode care must be given to choose a source with a reasonably low work function that is stable at elevated temperatures. This talk will focus on the pros and cons of past, present and future FE sources that span the CFE and SE continuum. Special emphasis will be given to the 
popular $\mathrm{Zr} / \mathrm{O} / \mathrm{W}$ source whose emission distribution is shown in figure 3 . The work function localization to the (100) plane allows for high axial brightness for $\mathrm{W}$ sources with a (100) crystal orientation. Recent advances in the prospects of carbon nanotubes as a FE source will also be discussed.

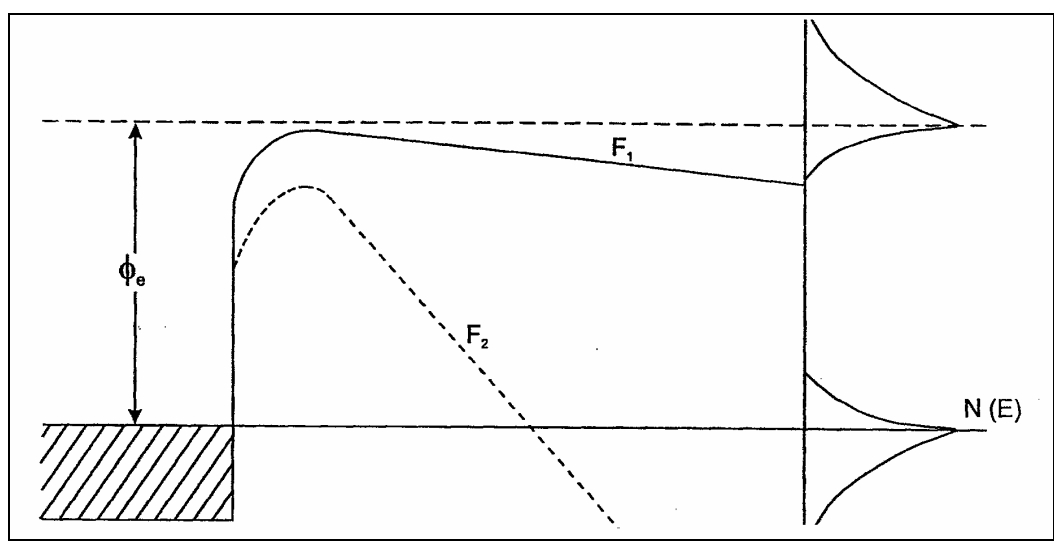

FIG. 1 Potential energy diagram illustrating CFE and SE emission extremes.

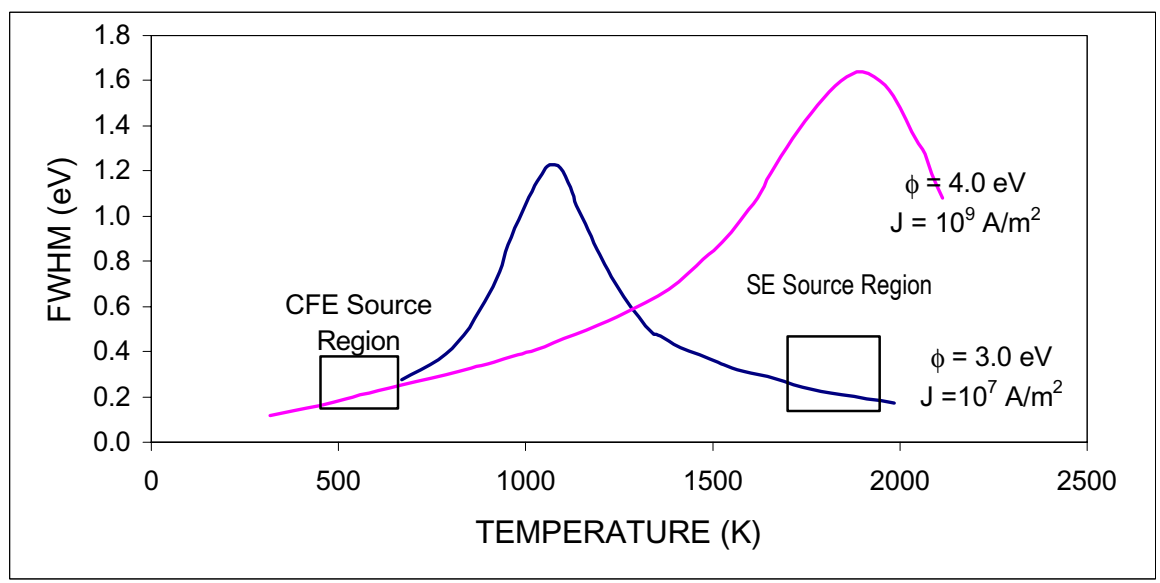

FIG. 2 Theoretical variation of the FWHM vs. source temperature at constant J.

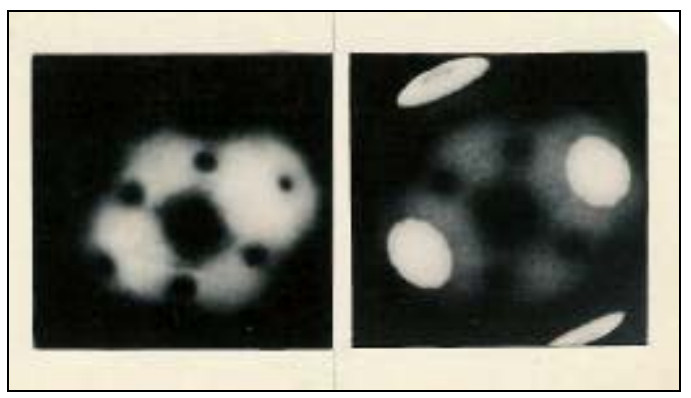

FIG. 3 The photo on the left shows the emission distribution of a clean W source. The right hand photo shows the selective work function lowering of the (100) planes upon application of $\mathrm{ZrO}_{2}$. 